\title{
Gain Stabilization in Gain Clamped EDFA Cascades Fed by WDM Burst-Mode Packet Traffic
}

\author{
Miroslav Karásek, Alberto Bononi, Member, IEEE, Leslie A. Rusch, Member, IEEE, Member, OSA, and \\ Mourad Menif, Student Member, IEEE
}

\begin{abstract}
This paper studies via simulation the stabilizing effect of all-optical gain-clamping (AOGC) in a chain of erbium-doped fiber amplifiers (EDFA) fed by wavelength-division multiplexing (WDM) burst-mode packet traffic. AOGC is necessary to suppress swings of output power and optical signal-to-noise ratio (OSNR). A case study is selected, in which only the first EDFA in a cascade of six amplifiers is clamped using a ring laser configuration. A numerical model which solves the transcendental equation for the average inversion at each EDFA is used for the analysis. The traffic is generated on the eight WDM channels by ON-OFF time-slotted sources, with statistically independent $O N$ and OFF durations, randomly generated by a truncated Pareto distribution with infinite variance. The simulation model includes the generation of amplified spontaneous emission within each amplifier and the propagation of the lasing power generated in the AOGC EDFA through the cascade. It is shown that the sizable power and OSNR swings arising in an unclamped cascade of EDFA's can be effectively suppressed when a lasing signal a few decibels above the aggregate signal power develops in the AOGC EDFA and propagates along the cascade.
\end{abstract}

Index Terms-Modeling, optical communication, optical fiber amplifiers, transient analysis, wavelength-division multiplexing (WDM).

\section{INTRODUCTION}

$\mathbf{T}$ HE capacity of current optical communications systems is limited by the use of electronics for signal generation, packet routing and buffering at intermediate switching nodes. Wavelength-division multiplexing (WDM) technology employing erbium-doped fiber amplifiers (EDFA's) provides a platform for significant improvement in network bandwidth capacity and WDM will play a dominant role in backbone infrastructure supporting the next generation high-speed networks. Fast signal power transients caused by cross-gain saturation effects pose a serious limitation in amplified WDM transmission networks. Whereas most previous analysis on cross-gain saturation in EDFA's focuses on circuit-switched scenarios, we address links carrying data packets. Thus our

Manuscript received August 16, 1999

M. Karásek is with the Centre for Optics, Photonics and Lasers (COPL), the Department of Electrical and Computer Engineering, Laval University, P.Q. G1K 7P4, Canada, on leave from the Institute of Radio Engineering and Electronics, Academy of Sciences of the Czech Republic, Chaberska 57, 18251 Prague, Czech Republic.

A. Bononi is with the Dipartimento di Ignegneria dell'Informazione, Universitá di Parma, Parma, I-43100, Italy.

L. A. Rusch and M. Menif are with Centre for Optics, Photonics and Lasers (COPL), the Department of Electrical and Computer Engineering, Laval University, P.Q. G1K 7P4, Canada.

Publisher Item Identifier S 0733-8724(00)02200-3. analysis applies to point-to-point links carrying internet protocol (IP) or other packet data, as well as systems using packet switching. Rapid growth of packet networks is expeceted due to the efficiency of internet protocol in servicing bursty traffic from computer users.

In WDM circuit-switching networks, the number of channels present in an EDFA may vary due to network reconfiguration or channel failure. This leads to cross-gain saturation in fiber amplifiers that in turn induce power transients in the surviving channels which can cause severe service impairment due to either inadequate eye opening or the appearance of optical nonlinearities [1]-[3]. A variety of methods have been proposed and demonstrated experimentally to prevent unwanted power excursions caused by channel addition/removal in concatenated fiber amplifiers. These include all-optical gain clamping (AOGC) via the construction of a ring laser [4], [5] or lasers with resonators defined by fiber Bragg gratings [6], or introduction of a control channel before the first optical amplifier in a link [7].

Even more serious bit error rate deterioration can arise in WDM packet-switching burst-mode networks, where no power is transmitted on empty slots. It has been shown that the high variability of ON-OFF times in packetized links may lead to self-similarity in the aggregate traffic [8], [9]. Long empty slot intervals may give enough time to fiber amplifiers to reach gains greatly exceeding the average values, which in turn leads to significant variation in output power and noise figure. This effect accumulates along a cascade of fiber amplifiers in the same way as the fast power transients in the circuit-switching scenario. Although the effect of traffic statistics on the output power and optical signal-to-noise ratio (OSNR) swings has been thoroughly investigated [10], [11], no measures have been suggested to minimize the variation in these quantities.

In this paper, we study the suppression of the spread in both the output power and OSNR due to variability of WDM asynchronous traffic at the input of a cascade of six EDFA's. Suppression is achieved by clamping the gain of the first amplifier using a ring laser configuration and propagating the lasing power through the cascade. The analysis is based on the application of a numerical model that solves the transcendental equation for the total number of excited erbium atoms in the doped fiber of each amplifier. This quantity, referred to as the reservoir is used as an initial condition for the subsequent time domain analysis. An iterative solution is applied in the AOGC EDFA to develop the lasing power from the amplified spontaneous emission (ASE). The transient analysis is based on the assumption that the atomic populations remain constant during a time step 
$\delta t$ of several hundreds of nanoseconds so that a direct time evolution of signal and ASE powers can be performed.

\section{Numerical Model of AOGC EDFA CASCADE}

To demonstrate the suppression of cross-gain saturation in concatenated EDFA's with the aid of all-optical gain clamping, we will investigate the time-dependent output power and OSNR fluctuations in a cascade of six amplifiers (shown schematically in Fig. 1) for a multiwavelength signal consisting of eight channels ranging from 1545 to $1552 \mathrm{~nm}$, with $1 \mathrm{~nm}$ spacing. The amplifiers are concatenated with transmission fibers of span loss $L_{k}$, where $k=1,2, \ldots, 6$ is the position of the transmission span and the fiber amplifier in the cascade.

The eight $\mathrm{ON}-\mathrm{OFF}$ sources simulating the time-slotted burst-mode WDM traffic were randomly generated in a way similar to that described in [11]. Packets fitting time slots of $2.8 \mu \mathrm{s}$, corresponding for instance to either asynchronous transfer mode (ATM) packets of about 500 bits at $155 \mathrm{Mb} / \mathrm{s}$, or IP segments of 4 kbytes at $10 \mathrm{~Gb} / \mathrm{s}$, were sampled at 10 points per packet. The resultant time step for the evolution of the reservoirs was $\delta t=0.3 \mu \mathrm{s}$. Simulation of the highly variable input traffic was performed over ten million time steps, corresponding to $3 \mathrm{~s}$ of transmission. Using a random number $U$ uniformly distributed on $[0,1]$, statistically independent ON and OFF intervals (in units of slots) were generated with a truncated Pareto distribution via

$$
T_{\mathrm{ON}}=\left\lfloor\frac{1}{U^{1 / \alpha_{\mathrm{ON}}}}\right\rfloor, \quad T_{\mathrm{OFF}}=\left\lfloor\frac{1}{U^{1 / \alpha_{\mathrm{off}}}}\right\rfloor
$$

where $\alpha_{\mathrm{OFF}}$ and $\alpha_{\mathrm{ON}}$ are parameters regulating the burstiness of the traffic, and $\lfloor x\rfloor$ is the floor function.

Our analysis is based on the application of a simplified numerical model which calculates the time evolution of the total number of excited erbium atoms in the doped fiber of each amplifier. This quantity, referred to as the reservoir [12], is expressed as:

$$
r(t)=r_{\max } \overrightarrow{\mathcal{N}}(t)
$$

where $r_{\max }=\rho A_{\text {eff }} \ell$ is the total number of erbium atoms in the amplifier, $\rho$ being the erbium concentration $\left[\mathrm{m}^{-3}\right], A_{\mathrm{eff}}$ the effective area of the doped part of fiber core $\left[\mathrm{m}^{2}\right]$, and $\ell$ the doped fiber length [m]; $\overline{\mathcal{N}}(t)=(1 / \ell) \quad \int_{0}^{\ell} \mathcal{N}_{2}(z, t) d z$ is the average inversion, $\mathcal{N}_{2}(z, t)$ being the fraction of erbium atoms in the excited state at coordinate $z$ and time $t$, averaged over the fiber cross section.

The model is based on a homogeneously broadened two-level approximation of an erbium ion and takes into account the propagation of signal, pump and both forward and backward ASE powers along the cascade of EDFA's. The wavelength axis is spectrally resolved into $M$ bins of constant width $\Delta \lambda$ spanning the range over which pump, signals and ASE are significant. Let $\mathcal{A}$ denote the set of all ASE bins, and $\mathcal{S}$ denote the set of bins on which the WDM signals, the gain stabilizing lasing power and the pump fall (the bin width is small enough such that at most one signal per bin is present, located at its center). The

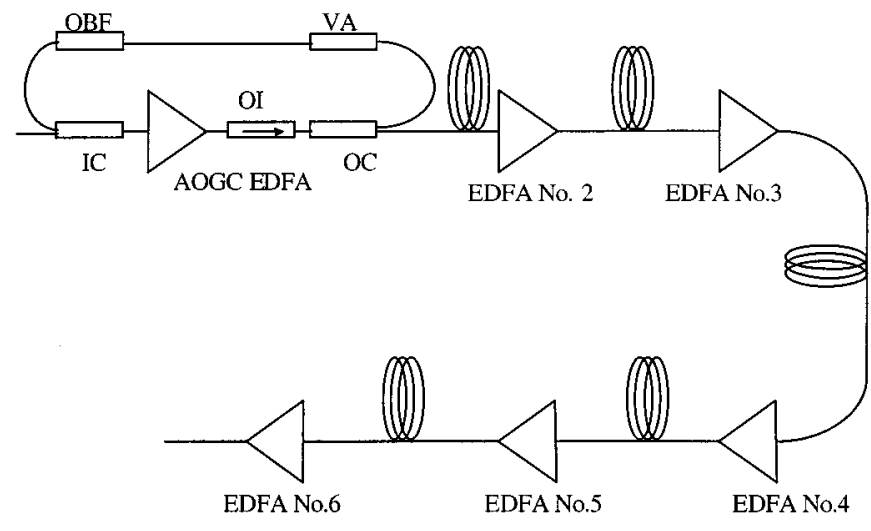

Fig. 1. Schematic diagram of an AOGC EDFA cascade: OBF: optical bandpass filter, OI: optical isolator, VA: variable attenuator, and IC, OC: wavelength insensitive input and output couplers.

equation describing the time evolution of the reservoir $r_{k}(t)$ at EDFA number $k$ is given by [12]

$$
\begin{aligned}
\frac{d}{d t} r_{k}(t)= & \frac{r_{k}(t)}{\tau}+\sum_{i \in\{\mathcal{S}, \mathcal{A}\}} Q_{i, k}^{\mathrm{in}}\left[1-G_{i, k}\left[r_{k}(t)\right]\right] \\
& -\sum_{i \in \mathcal{A}} 4 n_{i, k}^{s p}\left[r_{k}(t)\right]\left[G_{i, k}\left[r_{k}(t)\right]-1\right] \Delta \nu_{i}
\end{aligned}
$$

where:

- $\tau$ is the spontaneous lifetime of the excited level;

- $Q_{i, k}^{\text {in }}$ is the signal or pump (if $i \in \mathcal{S}$ ) or external ASE photon flux (if $i \in \mathcal{A}$ ) [ph/s] entering doped fiber $k$ at wavelength $\lambda_{i}$ corresponding to wavelength bin $i$. Signal fluxes are distinguished from ASE fluxes even when occupying the same wavelength bin, in order to be able to evaluate the OSNR at the EDFA output;

- $G_{i, k}\left[r_{k}(t)\right]=e^{B_{i, k} r_{k}(t)-A_{i, k}}$ is the gain at $\lambda_{i}$ of EDFA $k$, where $A_{i, k}, B_{i, k}$ are nondimensional coefficients, dependent on frequency $\nu_{i}$ through the erbium ions absorption and emission cross sections

$$
A_{i, k} \triangleq \alpha_{i} \ell_{k}, \quad B_{i, k} \triangleq\left(g_{i}+\alpha_{i}\right) /\left(\rho A_{\mathrm{eff}}\right),
$$

and $\alpha_{i} \triangleq \rho \Gamma_{i} \sigma_{i}^{a}$ and $g_{i} \triangleq \rho \Gamma_{i} \sigma_{i}^{e}$ are the absorption and emission constants; $l_{k}$ is the length of the $k$ th doped fiber; $\Gamma_{i}$ is the confinement factor at $\lambda_{i}$ calculated by the overlap integral between the radial distribution of the $\mathrm{LP}_{01}$ mode intensity and the erbium ions doping distribution; $\sigma_{i}^{a}$ and $\sigma_{i}^{e}\left[\mathrm{~m}^{2}\right]$ are the absorption and emission cross sections at $\lambda_{i}$, respectively;

- $n_{i, k}^{s p}\left[r_{k}(t)\right]=\left(g_{i} r_{k}(t)\right) /\left(\left(g_{i}+\alpha_{i}\right) r_{k}(t)-\alpha_{i} r_{\max }\right)$ is the spontaneous emission factor, and the summation in which it appears in (3) represents the ASE generated inside EDFA $k$, the factor 4 representing two polarization components for both forward and backward ASE; $\Delta \nu_{i}$ is the frequency width $[\mathrm{Hz}]$ of wavelength bin $i$.

The signal and ASE fluxes at the output of EDFA $k$ are

$$
\begin{aligned}
Q_{i, k}^{\text {out }}(t)= & Q_{i, k}^{\text {in }}(t) G_{i, k}\left[r_{k}(t)\right] F_{i, k} \quad i \in \mathcal{S} \\
Q_{i, k}^{\text {out }}(t)= & {\left[Q_{i, k}^{\text {in }}(t) G_{i, k}\left[r_{k}(t)\right]+2 n_{i, k}^{s p}(t)\right.} \\
& \left.\cdot\left(G_{i, k}\left[r_{k}(t)\right]-1\right) \Delta \nu_{i}\right] F_{i, k} \quad i \in \mathcal{A}
\end{aligned}
$$




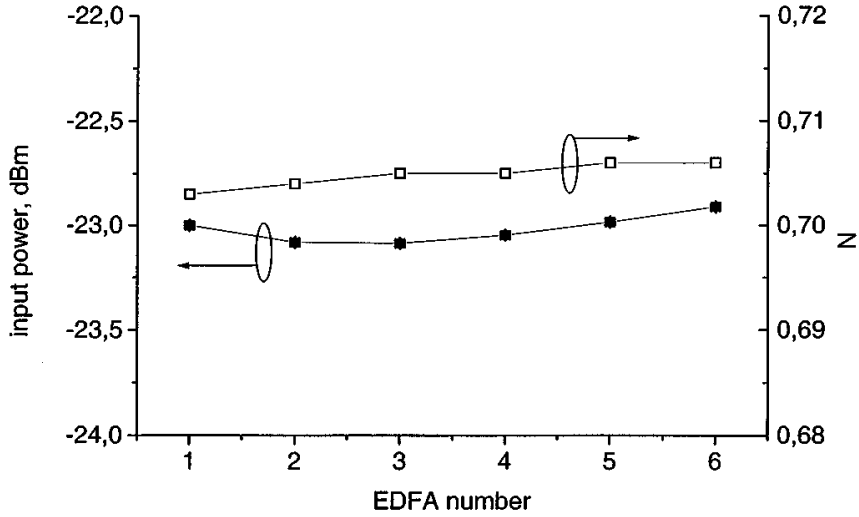

(a)

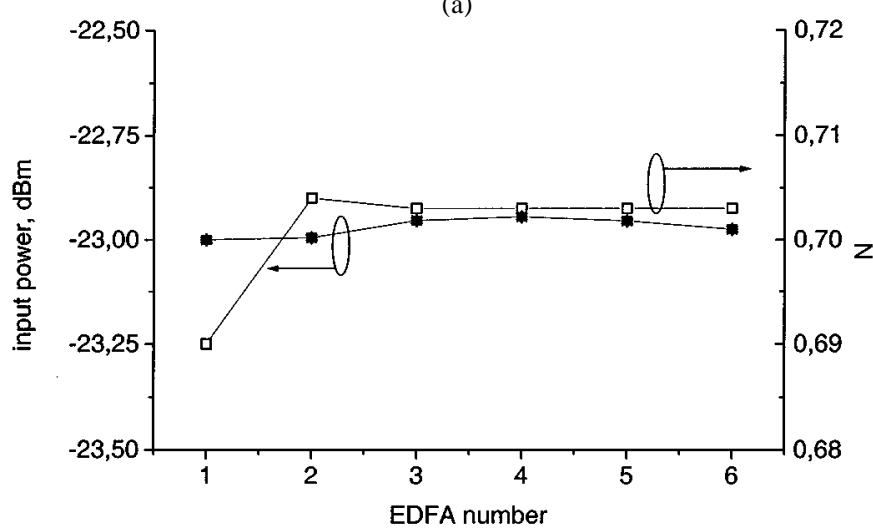

(b)

Fig. 2. (a) Evolution of input signal power and length averaged metastable level population density along the unclamped cascade A for CW input. (b) Evolution of input signal power and length averaged metastable level population density along the gain-clamped cascade $\mathrm{B}$ for $\mathrm{CW}$ input.

where $F_{i, k}$ is the power transmission of the gain equalizing filter $k$ at wavelength $\lambda_{i}$. We note in the above equation that only half of the ASE generated in the EDFA, corresponding to forward ASE, is present at the output of the EDFA and will be propagated downstream. Finally, indicating with $L_{k}$ the loss of the transmission span between EDFA's number $k$ and $k+1$, the updated fluxes at the input of doped fiber $k+1$ are

$$
\begin{aligned}
& Q_{i, k}^{\text {in }}(t)=Q_{i, k-1}^{\text {out }} C^{\text {out }} L_{k-1}, \quad k=2 \quad i \in\{\mathcal{S}, \mathcal{A}\} \\
& Q_{i, k}^{\text {in }}(t)=Q_{i, k-1}^{\text {out }} L_{k-1}, \quad k=3,4, \cdots 6 \quad i \in\{\mathcal{S}, \mathcal{A}\}
\end{aligned}
$$

Here $C^{\text {out }}$ represents the coupling ratio of the output coupler (OC). The equations describing the optical feedback and characterizing the input flux of the AOGC EDFA at each wavelength bin $i \in\{\mathcal{S}, \mathcal{A}\}$ are

$$
\begin{aligned}
Q_{i, 1}^{\text {in }}(t)= & Q_{s i g, i}^{\text {in }}(t) C^{\text {in }}+Q_{i, 1}^{\text {out }}(t)\left(1-C^{\text {out }}\right) \\
& \cdot T_{i} \beta\left(1-C^{\text {in }}\right) \quad i \in \mathcal{S} \\
Q_{i, 1}^{\text {in }}(t)= & Q_{i, 1}^{\text {out }}(t)\left(1-C^{\text {out }}\right) T_{i} \beta\left(1-C^{\text {in }}\right) \quad i \in \mathcal{A} .
\end{aligned}
$$

In these equations, $Q_{s i g, i}^{\text {in }}(t)$ is an external photon flux representing an WDM signal entering the cascade of EDFA's at wavelength $\lambda_{i}, C^{\text {in }}$ the coupling ratio of the input coupler (IC), $T_{i}$ is power transmission of the optical bandpass filter (OBF) at wavelength $\lambda_{i}$, and $\beta$ is the attenuation of the variable attenuator (VA). The optical bandpass filter effectively suppresses most of the ASE components fed back from the output to the input of the AOGC EDFA so that a lasing action occurs with $Q_{\text {las }}^{\text {in }}\left(\lambda_{l a s}\right)$ being the lasing photon flux at the input of EDF number 1 . For the solution of the reservoir equations at EDFA's $2, \ldots, 6$ the laser flux is treated as an external signal flux in (3).

Numerical solution to the time dependent equation (2) is separated into two steps: determination of a steady state value of $r_{k}$ for each EDFA in the cascade for continuous wave (CW) input signals, followed by a time evolution with burst-mode WDM traffic. To obtain a steady state value of $r_{k}$, a transcendental equation derived from (3) by setting $d r_{k}(t) / d t=0$ is solved numerically for $k=1,2, \ldots, 6$ starting with amplifier 1 which is gain clamped. In the case of the gain clamped EDFA, the transcendental equation is more complicated due to the optical feedback loop implemented in this amplifier. In addition to the state variable $r_{1}$, the laser flux $Q_{\text {las }}^{\text {in }}$ has to be found. An iterative solution was used to solve the transcendental equation for the steady state value of $r_{1}$, while the transcendental equations for steady-state $r_{k}, k=2, \ldots, 6$ are solved directly.

\section{RESULTS}

In this analysis, the ASE is considered over the spectral region from 1450 to $1650 \mathrm{~nm}$, subdivided into $M=200$ bins of width $\delta \lambda=1 \mathrm{~nm}$. We consider a typical Lucent Technologies EDF pumped at $1480 \mathrm{~nm}$. The coupling ratios of the input and the output couplers were assumed to be $C^{\text {in }}=0.9, C^{\text {out }}=0.5$, respectively. We assume that each amplifier in the cascade has a gain equalization filter that perfectly equalizes the gain over the signals and lasing wavelength range [13]. The amplifiers are designed to have a gain of $20 \mathrm{~dB}$ at the continuous-wave (CW) input signal power of $-23 \mathrm{dBm}$ per channel to compensate for the span loss of $L_{k}=20 \mathrm{~dB}, k=1,2, \ldots, 5$. In order to demonstrate the suppression of output power and OSNR swings due to cross-gain saturation effects, performance of an AOGC cascade and an unclamped cascade will be compared

- cascade A: no AOGC present; EDFA lengths $\ell_{1}-\ell_{6}=$ $24.5 \mathrm{~m}$; pump powers $P_{p 1}-P_{p 6}=18.5,19,19.5,20$, 20.5 , and $21 \mathrm{~mW}$

- cascade B: AOGC present at EDFA number 1; EDFA lengths of $\ell_{1}=30 \mathrm{~m}, \ell_{2}-\ell_{6}=25 \mathrm{~m}$, pump powers of $P_{p 1}=60 \mathrm{~mW}, P_{p 2}-P_{p 6}=49.5 \mathrm{~mW}$, respectively.

The lasing wavelength was set at $\lambda_{\text {las }}=1558 \mathrm{~nm}$. The total ring resonator loss was adjusted to $23.3 \mathrm{~dB}$ which gives the lasing power after the output coupler $P_{\text {las }}^{\text {out }}=10 \mathrm{~mW}$. The gain of the AOGC EDFA is $23 \mathrm{~dB}, 3 \mathrm{~dB}$ higher than that of EDFA's $2, \ldots 6$, to compensate for the additional loss of the output coupler (OC) and the following span loss $L_{1}=20 \mathrm{~dB}$.

We will first show the steady-state characteristics for two cascades. Fig. 2(a) and (b) shows the evolution of the continuous wave input signal powers $P_{s i g, i}^{\text {in }}$ and the average inversion $\overline{\mathcal{N}}$ at steady state along cascades A and B, respectively. It is seen that, due to the gain equalization filters and the adjustment of pump powers of individual amplifiers, the signal power and $\overline{\mathcal{N}}$ are kept almost constant along both cascades. For the dynamic analysis, the power of each WDM channel is modulated according to an ON-OFF slotted renewal process. An infinite variance was chosen for the ON and OFF periods for each WDM 


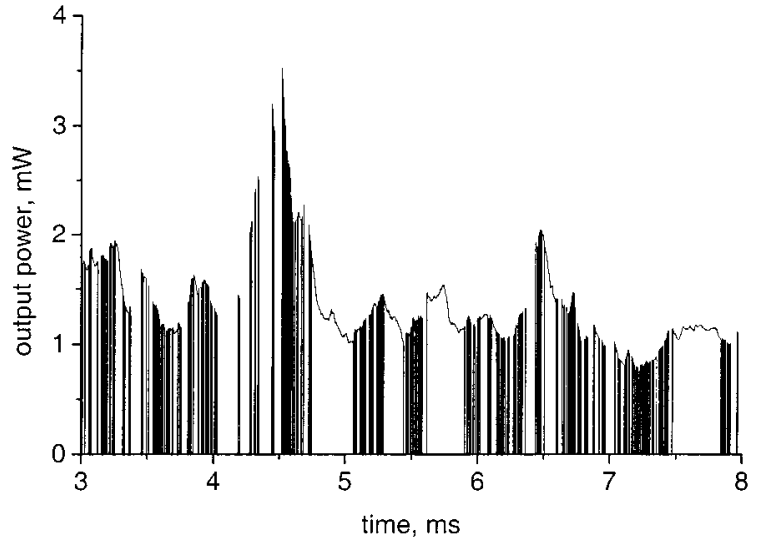

(a)

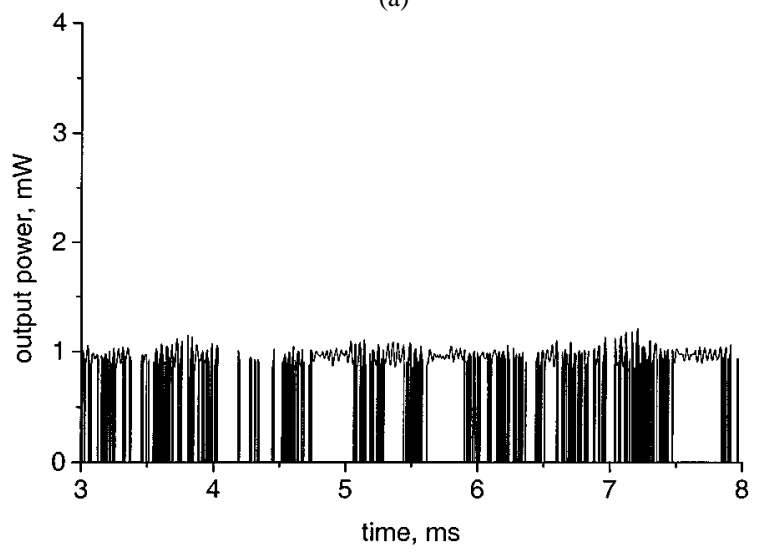

(b)

Fig. 3. (a) Time evolution of signal power at $\lambda_{s}=1551 \mathrm{~nm}$ at the output of EDFA number 6: unclamped cascade A. (b) Time evolution of signal power at $\lambda_{s}=1551 \mathrm{~nm}$ at the output of EDFA number 6: gain-clamped cascade B.

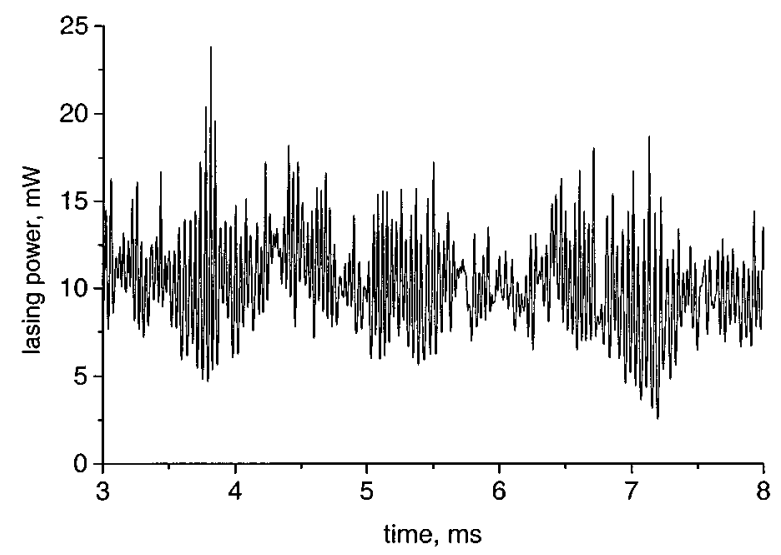

Fig. 4. Time evolution of gain stabilizing lasing power at $\lambda_{\text {las }}=1558 \mathrm{~nm}$ at the output of EDFA number 6: gain-clamped cascade B.

signal $\left(\alpha_{\mathrm{ON}}=\alpha_{\mathrm{OFF}}=1.2\right)$ [11]. The average utilization of each WDM channel is

$$
u=\frac{E\left[T_{\mathrm{ON}}\right]}{E\left[T_{\mathrm{ON}}\right]+E\left[T_{\mathrm{OFF}}\right]}
$$

where $E\left[T_{\mathrm{ON}}\right], E\left[T_{\mathrm{OFF}}\right]$ are the mean values of the ON and OFF periods. In our case, since these are equal, we have $u=0.5$. The input power per channel to the chain was increased by 3 $\mathrm{dB}$ with respect to the continuous wave analysis reported in the

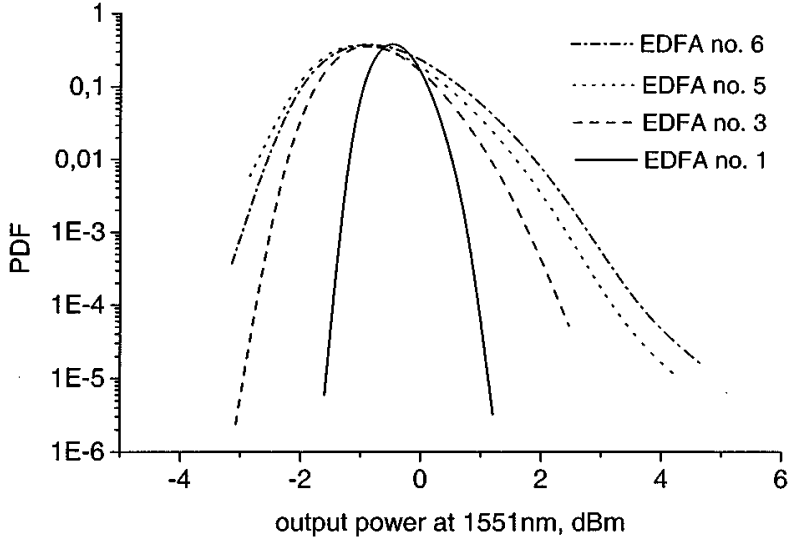

(a)

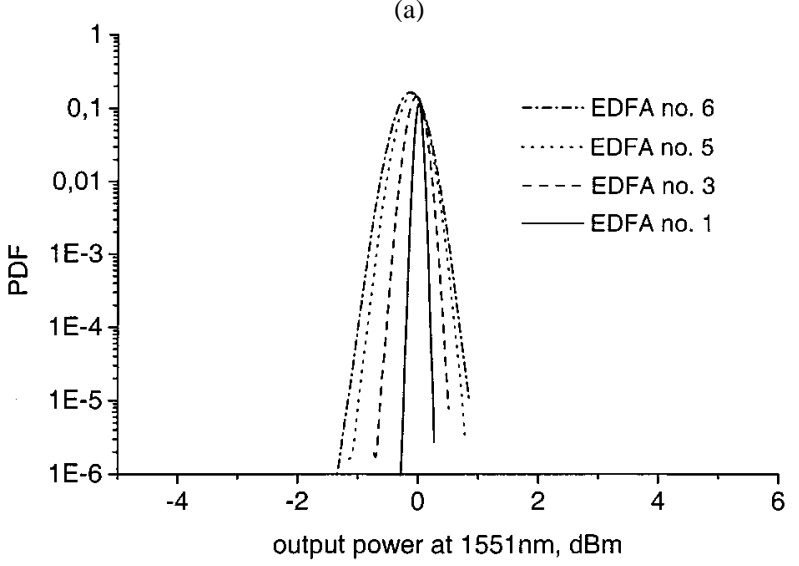

(b)

Fig. 5. (a) Probability density function of signal power at $\lambda=1551 \mathrm{~nm}$ at the output of amplifiers 1, 3, 5, and 6 of unclamped cascade A. (b) Probability density function of signal power at $\lambda=1551 \mathrm{~nm}$ at the output of amplifiers 1 , 3,5 , and 6 of gain-clamped cascade B.

previous figure, in order to keep the "dynamic average" gain equal to the span loss. Fig. 3(a) and (b) shows the time evolution of the output signal at channel 1 at $\lambda_{s}=1545 \mathrm{~nm}$ at the output of EDFA number 6 for cascades A and B, respectively, for a time period of $5 \mathrm{~ms}$. Significant power swings occur at the output of the unclamped cascade A as a result of cross-gain saturation in the fiber amplifiers and the highly variable traffic in the other channels. In the clamped cascade B the power fluctuations are substantially reduced due to the stabilization effect of the lasing power generated in the AOGC EDFA and propagating through the cascade $\mathrm{B}$. The time variation of the lasing power at the output of EDFA number 6 is plotted in Fig. 4. The lasing signal effectively "absorbs" the variablility on the input signals, allowing the flatter output observed in Fig. 3(b).

Histograms of output power and optical signal-to-noise ratio were evaluated at each EDFA for all eight channels, and approximate the probability density function for output power and OSNR. In Fig. 5(a) and (b) the histograms of signal power for channel number 7 at $1551 \mathrm{~nm}$ at the output of EDFA number 6 are plotted for cascades A and B, respectively. In both the unclamped and clamped case, the output power fluctuations grow along the cascade. Power spreads of 1.7, 3.4, 4.3, and $4.5 \mathrm{~dB}$ with probability larger than $10^{-2}$ were recorded for EDFA's $1,3,5$, and 6 , respectively, for the unclamped cascade A. The 


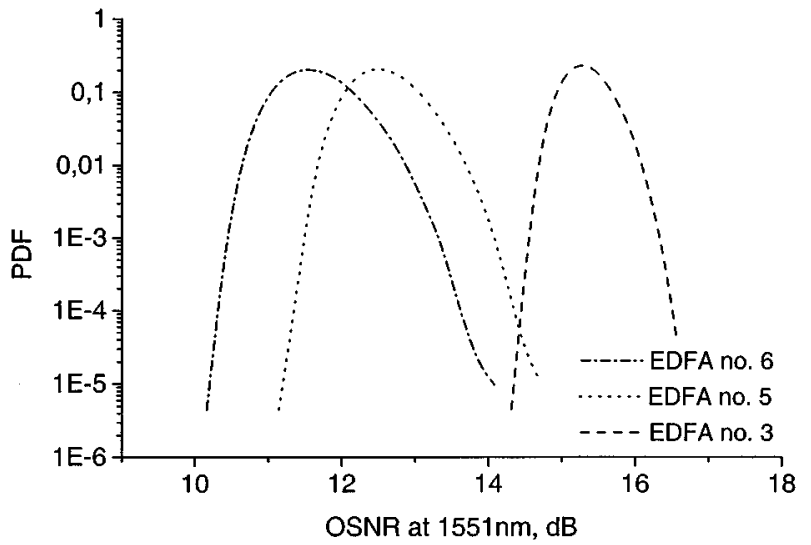

(a)

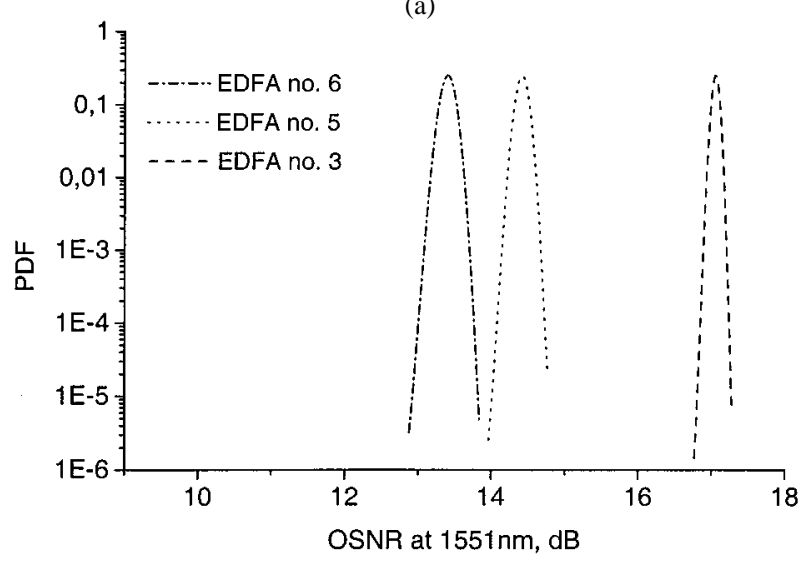

(b)

Fig. 6. (a) Probability density function of OSNR at $\lambda=1551 \mathrm{~nm}$ at the output of amplifiers 3, 5, and 6 of unclamped cascade A. (b) Probability density function of OSNR at $\lambda=1551 \mathrm{~nm}$ at the output of amplifiers 3, 5, and 6 of gain-clamped cascade $\mathrm{B}$

output power swings after amplifiers $1,3,5$, and 6 were reduced by more than one order of a magnitude to $0.24,0.42,0.80$, and $0.90 \mathrm{~dB}$ in the clamped cascade B. A similar reduction was observed in the spread of OSNR. The histograms of OSNR at $\lambda_{s}=1551 \mathrm{~nm}$ after amplifiers 3, 5, and 6 for the cascades A and B are given in Fig. 6(a) and (b). The range of OSNR fluctuations occuring with probability higher than $10^{-2}$ is equal to 1.4, 2.0, and $2.3 \mathrm{~dB}$ after EDFA's 3, 5, and 6, respectively, for the unclamped cascade $\mathrm{A}$ but it was reduced to $0.25,0.40$, and $0.46 \mathrm{~dB}$ after amplifiers 3,5 , and 6 , of the clamped cascade B.

We next investigate the effect of the level of the lasing power propagating through the gain-clamped cascade on the suppression of EDFA cross-gain saturation. In contrast to cascade B where $P_{\text {las }}^{\text {out }}=10 \mathrm{~mW}$, the lengths of the AOGC EDFA and EDFA's $2,3, \ldots, 6$ and their pump powers were selected to obtain $P_{\text {las }}^{\text {out }}=5 \mathrm{~mW}$ (cascade C) and $P_{\text {las }}^{\text {out }}=20 \mathrm{~mW}$ (cascade D). Gains of $23 \mathrm{~dB}$ for the AOGC EDFA and $20 \mathrm{~dB}$ for EDFA's $2,3, \ldots, 6$ were selected to simulate a balanced gain-clamped cascade as in the case of cascade B. The corresponding EDFA lengths and pump powers for these two cascades were as follows:

- cascade C: $P_{\text {las }}^{\text {out }}=5 \mathrm{~mW}, \ell_{1}=30 \mathrm{~m}, \ell_{2}-\ell_{6}=24 \mathrm{~m}$, pump powers of $P_{p 1}=40 \mathrm{~mW}, P_{p 2}-P_{p 6}=34.5 \mathrm{~mW}$;

- cascade D: $P_{\text {las }}^{\text {out }}=20 \mathrm{~mW}, \ell_{1}=31 \mathrm{~m}, \ell_{2}-\ell_{6}=27.5$ m, pump powers of $P_{p 1}=90 \mathrm{~mW}, P_{p 2}-P_{p 6}=61 \mathrm{~mW}$.

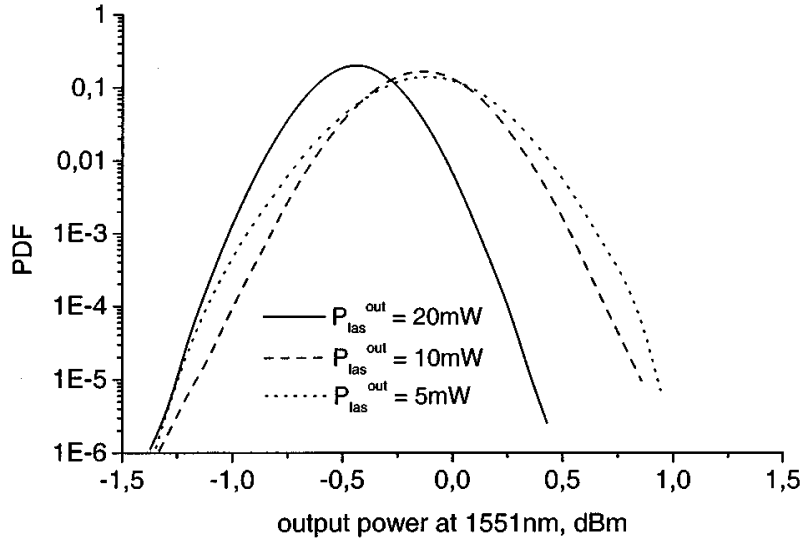

(a)

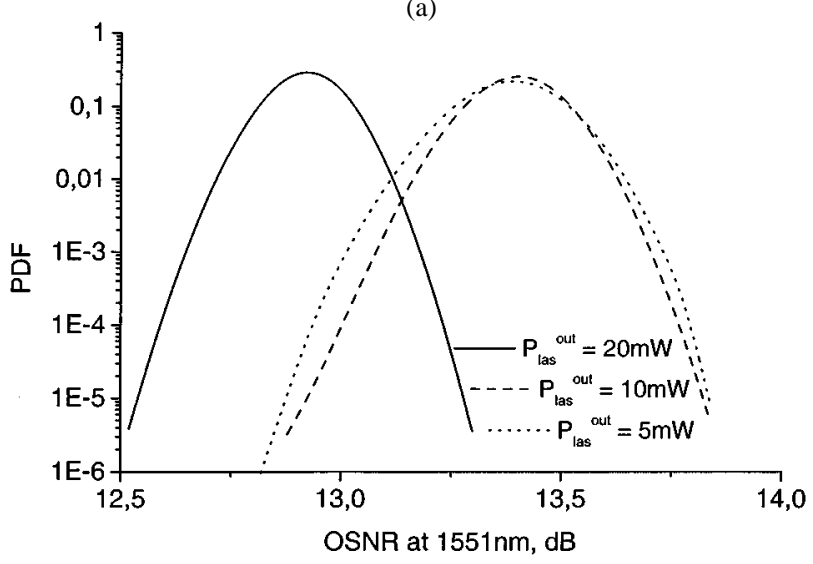

(b)

Fig. 7. (a) Probability density function of output power at $\lambda=1551 \mathrm{~nm}$ at the output of gain-clamped cascades C, B, and D. (b) Probability density function of OSNR at $\lambda=1551 \mathrm{~nm}$ at the output of gain-clamped cascades C, B, and D.

Fig. 7(a) and (b) shows the output power and OSNR histograms for channel number 7 at $\lambda_{s}=1551 \mathrm{~nm}$ at the output of cascade $\mathrm{B}, \mathrm{C}$, and $\mathrm{D}$. The range of output power fluctuations occuring with probability higher than $10^{-2}$ is equal to $1.2,0.9$, and 0.80 $\mathrm{dB}$ for $P_{\text {las }}^{\text {out }}=5,10$, and $20 \mathrm{~mW}$, respectively. Signal-to-noise ratio swings occuring with probability higher than $10^{-2}$ at the output of cascades C, B, and D are $0.53,0.46$, and $0.39 \mathrm{~dB}$, respectively. In comparison with the unclamped cascade A, output power and OSNR swings caused by EDFA cross-gain saturation and a highly variable packetized traffic may be reduced by a factor of 6 if the lasing power at the output of the clamped EDFA's is kept at the level of $13 \mathrm{dBm}$, i.e., only $4 \mathrm{~dB}$ larger than the aggregate signal power. The price of the gain stabilization effect is a substantially larger pump power necessary to provide the gain equal to the span loss of $20 \mathrm{~dB}$.

\section{CONCLUSION}

Our analysis is based on a theoretical model incorporating time variation effects and propagation of ASE in an EDFA, including solution of a transcendental equation describing the length averaged metastable level population density. We investigated the effect of clamping the gain of the first amplifier in the cascade by implementing a ring laser and propagating the lasing power through the cascade on the statistics of output power and 
signal-to-noise ratio variation in an eight channel WDM cascade of six amplifiers. The input traffic on each channel was modeled as a succession of periods of transmission and silence (ON periods and OFF periods) of packetized data. The ON periods and OFF periods were modeled as independent and each having a heavy-tailed Pareto distribution exhibiting self-similar behavior. In order to capture the heavy tails of the output power and noise figure swings, we simulated over 10 million time slots.

Our simulation results demonstrate that swings in both the output power and the OSNR can be substantially reduced when a sufficient amount of lasing power is allowed to develop in the gain-clamped EDFA and propagated through the cascade. In comparison with an unclamped cascade of six conventional EDFA's, the variation in output power and OSNR at the probability level of $10^{-2}$ at the end of a gain-clamped cascade is reduced by a factor of six if the lasing power is only $4 \mathrm{~dB}$ above the aggregate signal power. The price of the gain stabilization effect is a higher pump power necessary to keep the gain of individual amplifiers equal to the span loss of $20 \mathrm{~dB}$, even when the EDFA's are saturated by the propagating lasing power.

\section{REFERENCES}

[1] A. K. Srivastava, Y. Sun, J. L. Zyskind, J. W. Sulhoff, C. Wolf, and R. W. Tkach, "Fast gain control in an erbium-doped fiber amplifier," in Proc. Optic. Amp. Appl., Monterey, CA, 1996, p. paper PDP4.

[2] A. K. Srivastava, Y. Sun, J. L. Zyskind, and J. W. Sulhoff, "EDFA transient response to channel loss in WDM transmission system," IEEE Photon. Technol. Lett., vol. 9, pp. 386-388, 1997.

[3] J. L. Zyskind, A. K. Srivastava, Y. Sun, J. C. Ellson, G. W. Newsome, R. W. Tkach, A. R. Chraplyvy, J. W. Sulhoff, T. A. Strasser, J. R. Pedrazzani, and C. Wolf, "Fast link control protectio for surviving channels in multiwavelength otical networks," in Proc. Eur. Conf. Opt. Commun., Oslo, Norway, 1996, p. paper ThC.3.6.

[4] H. Dai, J. Y. Pan, and C. Lin, "All-optical gain control of in-line erbiumdoped fiber amplifiers for hybrid analog/digital WDM systems," IEEE Photon. Technol. Lett., vol. 9, pp. 737-739, 1997.

[5] D. H. Richards, J. L. Jackel, and M. A. Ali, "Multichannel EDFA chain control: A comparison of two all-optical approaches," IEEE Photon. Technol. Lett., vol. 10, pp. 156-158, 1998.

[6] J. F. Massicott, S. D. Willson, R. Wyatt, J. R. Armitage, R. Kashyap, and D. Williams, "1480 nm pumped erbium doped fiber amplifiere with all-optical automatic gain control," Electron. Lett., vol. 30, pp. 962-964, 1994.

[7] J. L. Zyskind, A. K. Srivastava, Y. Sun, J. C. Ellson, G. W. Newsome, R. W. Tkach, A. R. Chraplyvy, J. W. Sulhoff, T. A. Strasser, J. R. Pedrazzani, and C. Wolf, "Fast link control protection for surviving channels in multiwavelength otical networks," in Proc. Eur. Conf. Opt. Commun., Oslo, Norway, 1996, p. Paper ThC.3.6.

[8] W. E. Leland, M. S. Taqqu, W. Wilinger, and D. V. Wilson, "On the self-similar nature of ethernet traffic (extended version)," IEEE/ACM Trans. Networking, vol. 2, pp. 1-15, 1994.

[9] J. Beran, R. Sherman, M. S. Taqqu, and D. V. Wilinger, "(1)ong-range dependence in variable-bit-rate video traffic," IEEE Trans. Commun., vol. 43, pp. 1566-1579, 1995

[10] A. Bononi, L. Tanc evski, and L. A. Rusch, "Large power swings in doped-fiber amplifiers with highly variable data," IEEE Photon. Technol. Lett., vol. 10, pp. 131-133, 1998.
[11] L. Tanc evski, A. Bononi, and L. A. Rusch, "Output power and SNR swings in cascades of EDFA's for circuit- and packet-switched optical networks," J. Lightwave Technol., vol. 17, pp. 733-742, 1999.

[12] A. Bonini and L. A. Rusch, "Doped-fiber amplifier dynamics: A system perspective," J. Lightwave Technol., vol. 16, pp. 945-956, 1998.

[13] M. Rochette, M. Guy, S. LaRochelle, J. Lauzon, and F. Trépanier, "Gain equalization of EDFAs' with Bragg gratings," IEEE Photon. Technol. Lett., vol. 11, pp. 536-538, 1999.

Miroslav Karásek received the Ing. degree with honors from Prague Technical University, Czechoslovakia, in 1969 and the Ph.D. degree in microwave semiconductor devices from the Institute of Radioengineering and Electronics (IREE), Czechoslovak Academy of Sciences, Prague, in 1974.

$\mathrm{He}$ is a Senior Researcher at IREE, Academy of Sciences of the Czech Republic. Currently, he is a Visiting Researcher at the Centre for Optics, Photonics and Lasers (COPL), the Department of Electrical and Computer Engineering, Université Laval, PQ, Canada. His research activities are in the area of measurement and computer modeling of active fibers.

Alberto Bononi (S'92-M'95) received the "Laurea in Ingegnería Elettronica" (cum laude) from the University of Pisa, Pisa, Italy, in 1988 and the M.A. and Ph.D. degrees in electrical engineering from Princeton University, Princeton, NJ, in 1992 and 1994, respectively.

From 1994 to 1996, he was an Assistant Professor in Electrical and Computer Engineering Department at the State University of New York at Buffalo (SUNY), Buffalo, NY, teaching courses on circuits and systems, and on optical networks. Since 1996, he has been an Assistant Professor at the University of Parma, Italy, where he teaches courses on communications networks, probability theory, and stochastic processes. His main research interests include system design and performance issues in fast packet switching and high-speed all-optical networks, nonlinear fiber transmission design for WDM systems, and gain dynamics in fiber amplifier chains. He has been the Scientific Coordinator of the European Community sponsored project DAWRON on wavelength-routed optical networks.

Leslie A. Rusch (S'91-M'94) was born in Chicago, IL. She received the B.S.E.E. degree with honors from the California Institute of Technology, Pasadena, in 1980 and the M.A. and Ph.D. degrees in electrical engineering from Princeton University, Princeton, NJ, in 1992 and 1994, respectively.

She is currently an Assistant Professor in Department of Electrical and Computer Engineering at Université Laval, PQ, Canada. She occupies a chair in optical communications jointly sponsored by the Natural Science and Engineering Research Council of Canada and QuébecTel. Her research interests include fiber-optic communications, transient gain analysis of erbium-doped fiber amplifiers, wireless communications, spread-spectrum communications, and code division multiple access for radio and optical frequencies.

Dr. Rusch is a member of the Optical Society of America (OSA).

Mourad Menif (S'99) was born in Tunis on April 23, 1973. He received the B.S.E.E., M.S.E.E., and D.E.A. degrees from the Ecole Nationale d'Ingénieurs de Tunis. He is currently pursuing the Ph.D. degree in electrical engineering at the Université Laval, PQ, Canada, in the field of optical fiber communications.

His research interests include transient gain analysis of EDFA's fed by highspeed, self-similar, and heavy traffic for WDM long-haul and access networks. 